Nicholas B. Sajjadi*, BS, William Nowlin, BS, Ross Nowlin, BS, David Wenger, BS, John Martin Beal, DO, Matt Vassar, PhD and Micah Hartwell, PhD

\title{
United States internet searches for "infertility" following COVID-19 vaccine misinformation
}

https://doi.org/10.1515/jom-2021-0059

Received February 17, 2021; accepted March 22, 2021;

published online April 12, 2021

\begin{abstract}
Context: On December 1, 2020, Drs. Wolfgang Wodarg and Michael Yeadon petitioned to withhold emergency use authorization of the BNT162b2 messenger ribonucleic acid vaccine for coronavirus disease 2019 (COVID-19) manufactured by BioNTech and Pfizer, raising concern for female infertility risks but acknowledging the lack of evidence. The European Medicines Agency and the US Food and Drug Administration ultimately issued emergency use authorizations, but misinformation claiming that COVID-19 vaccines cause female infertility began circulating on social media, potentially influencing public perception and medical decision making among pregnant patients or those seeking to become pregnant.

Objectives: To determine the potential influence misinformation may have had on public interest in infertility related topics, as analyzed through internet search statistics in the US.
\end{abstract}

Methods: The Google Trends tool was used to analyze results for the search terms "infertility," "infertility AND vaccine," and "infertility AND COVID vaccine" in the US from February 4, 2020 to February 3, 2021. We applied

\footnotetext{
*Corresponding author: Nicholas B. Sajjadi, BS, Office of Medical Student Research, Oklahoma State University Center for Health Sciences, 1111 W 17th St., Tulsa, OK 74107-1886, USA,

E-mail: nicholas.sajjadi@okstate.edu. https://orcid.org/0000-00031716-2404

William Nowlin, BS, Ross Nowlin, BS and David Wenger, BS, Office of Medical Student Research, Oklahoma State University Center for Health Sciences, Tulsa, OK, USA

John Martin Beal, DO, Department of Obstetrics \& Gynecology, Ascension Oklahoma St. John Medical Center, Tulsa, OK, USA Matt Vassar, PhD and Micah Hartwell, PhD, Office of Medical Student Research, Oklahoma State University Center for Health Sciences, Tulsa, OK, USA; and Department of Psychiatry and Behavioral Sciences, Oklahoma State University Center for Health Sciences, Tulsa, OK, USA. https://orcid.org/0000-0001-6810-6571 (M. Hartwell)
}

autoregressive integrated moving average models to forecast expected values, comparing them with actual observed values.

Results: At peak interest (100), the forecasted relative search volumes interest for the search terms "infertility," "infertility AND vaccine," and "infertility AND COVID vaccine” were 45.47 (95\% CI, 33.27-57.66; $\mathrm{p}<0.001$ ), 0.88 (95\% CI, 2.87-4.63; $\mathrm{p}<0.001$ ), and 0.29 (95\% CI, -2.25-2.82; $\mathrm{p}<0.001)$. The actual relative search volumes at peak searching represented 119.9, 11,251, and 34,900\% increases, respectively, when compared with forecasted values.

Conclusions: COVID-19 vaccine misinformation corresponded with increased internet searches for topics related to infertility in the US. Dispelling misinformation and informing patients about the risks and benefits of COVID-19 vaccination may prevent unnecessary vaccine hesitancy or refusal, contributing to successful vaccination efforts.

Keywords: COVID-19; Google Trends; infertility; internet searches; patient information; vaccine.

Drs. Wolfgang Wodarg and Michael Yeadon submitted a petition to the European Medicines Agency (EMA) on December 1, 2020, requesting that emergency authorization for the BNT162b2 messenger ribonucleic acid (mRNA) coronavirus 2019 (COVID-19) vaccine manufactured by BioNTech and Pfizer be withheld pending further demonstration of safety and efficacy [1]. The petition claimed that the clinical trial design was inadequate and posed irreparable public harm [1]. Specifically, the petitioners raised concern for possible female infertility risks. The petitioners alleged that female infertility could arise secondary to vaccine induced antibodies against Syncytin-1, a mammalian placental protein that shares short homologous sequences with a severe acute respiratory syndrome (SARS) coronavirus spike protein [2]. Additionally, the Pfizer-BioNTech COVID-19 vaccine clinical trial did not include pregnant or lactating women, and safety and efficacy data among this population is lacking [3]. Though there may be biological merit for concerns about COVID-19 illness and pregnancy complications [4], the 
petitioners acknowledged the absence of any evidence for female infertility risks associated with COVID-19 vaccines [1].

The EMA ultimately issued emergency authorization on December 21, 2020, providing the first means of long term protection against COVID-19 transmission in the EU [5]. The US Food and Drug Administration also issued emergency authorization for the BNT162b2 COVID-19 vaccine [6] as well as the mRNA-1273 COVID-19 vaccine manufactured by Moderna [7] in December 2020. However, as distribution efforts began, misinformation stemming from inappropriate interpretations of the EMA petition by antivaccination campaigns began to circulate on social media platforms [8]. Misinformation claiming that COVID-19 vaccines cause female infertility without evidence presents a public health concern that may hinder successful vaccination efforts, especially among pregnant patients or those seeking to become pregnant. Thus, we sought to examine changes in public interest via internet searches for infertility and COVID-19 vaccines in the US after the spread of misinformation on social media.

\section{Methods}

The Oklahoma State University Institutional Review Board determined that this study did not qualify as human subjects research.

Google Trends (https://trends.google.com/trends/) is useful for identifying regional population interests and was used to monitor mental health concerns early in the COVID-19 pandemic [9]. We used the Google Trends tool on March 4, 2021 to examine internet traffic associated with the search term "infertility" in the US from February 4, 2020 through February 3, 2021. We used this date range to include the day the EMA petition was released (December 1, 2020), to build a strong model, and to avoid confounding from other events possibly related to infertility. We also included the combined search term "infertility AND vaccine" to capture baseline searches for infertility topics related to vaccines in general over the same time period, as well as the combined search term "infertility AND COVID vaccine" to quantify searches specifically related to infertility and COVID-19 vaccines.

We used autoregressive integrated moving average (ARIMA) models to forecast search behavior for these terms from December 1, 2020 onward, comparing actual values to projected values. Data extracted from Google Trends is presented in the form of relative search volume (RSV) ranging from $0-100$, with 100 representing peak searches for the specified region over the specified time period. All analyses were conducted in R Version 4.0.2 (R Core Team).

\section{Results}

Peak searches for "infertility" and "infertility and vaccine" occurred during the week of December 6, 2020, while peak searches for "infertility and COVID vaccine" occurred on December 13, 2020. The peak forecasted RSV for "infertility" was 45.47 (95\% confidence interval [CI], 33.27-57.66; $\mathrm{p}<0.001)$ compared with the actual RSV of 100, representing a 119.9\% increase in RSV compared with expected values. The peak forecasted RSV for "infertility and vaccine" was 0.88 (95\% CI, 2.87-4.63; p<0.001) compared with the actual RSV of 100, representing a 11,251\% increase in RSV compared with expected values. The peak forecasted RSV for "infertility and COVID vaccine" was 0.29 (95\% CI, -2.25-2.82; $\mathrm{p}<0.001)$ compared with the actual RSV of 100 , representing $34,900 \%$ increase in RSV compared to expected values (Figure 1). The actual RSV at peak searching (100) for each term was outside of the $95 \%$ CI generated by the ARIMA models.

Each term's actual RSV was higher than the forecasted values at 4 and 8 weeks from the EMA petition's release. The mean differences between each search term's actual and forecasted RSVs were statistically significant at 8 weeks (Table 1). RSV data for each term are listed at 4 week intervals over the course of our study period in Table 2 .

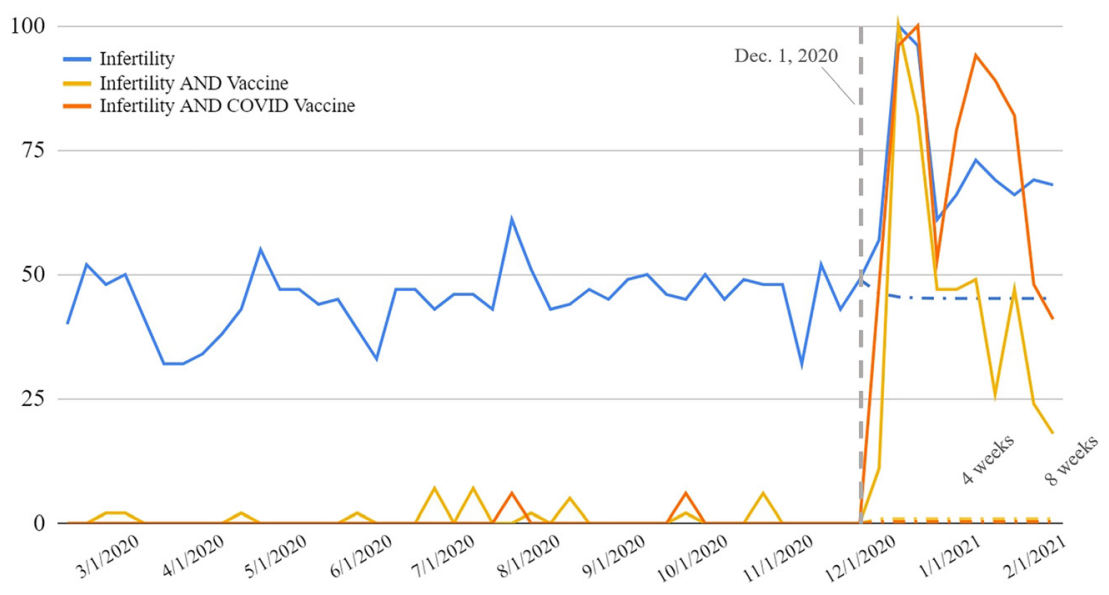

Figure 1: Actual relative search volumes for each term are designated by the colored solid lines.

The colored, dashed lines are the forecasted values from the autoregressive integrated moving average models for each term. The vertical dashed line on December 1, 2020 signifies the day the European Medicines Agency petition [1] was submitted and made publicly available. 
Table 1: Forecasted versus actual relative search volumes (RSV) for Google Trends terms at 4 and 8 weeks from December 1, 2020.

\begin{tabular}{|c|c|c|c|c|c|c|}
\hline \multirow[t]{2}{*}{ Search term } & \multicolumn{3}{|c|}{ Four weeks from December 1, 2020} & \multicolumn{3}{|c|}{ Eight weeks from December 1, 2020} \\
\hline & $\begin{array}{r}\text { Forecasted } \\
\text { RSV, mean (SD) }\end{array}$ & $\begin{array}{r}\text { Actual RSV, } \\
\text { mean (SD) }\end{array}$ & $t$, p-Value & $\begin{array}{r}\text { Forecasted RSV, } \\
\text { mean (SD) }\end{array}$ & $\begin{array}{r}\text { Actual RSV, } \\
\text { mean (SD) }\end{array}$ & $t$, p-Value \\
\hline Infertility & $45.54(0.47)$ & $78.5(22.63)$ & $-2.92,0.06$ & $45.36(0.36)$ & $73.50(15.90)$ & $-5.00,0.002$ \\
\hline Infertility and vaccine & $0.88(0.0)$ & $60.00(39.39)$ & $-3.00,0.06$ & $0.88(0.0)$ & $51.13(28.38)$ & $-5.00,0.002$ \\
\hline Infertility and 'COVID vaccine' & $0.29(0.0)$ & $74.25(27.55)$ & $-5.37,0.012$ & $0.29(0.0)$ & $80.13(19.61)$ & $-11.52,<0.0001$ \\
\hline
\end{tabular}

SD, standard deviation.

\section{Discussion}

Our findings suggest that circulating misinformation claiming COVID-19 vaccines cause female infertility corresponded with significant increases in internet searches for topics related to infertility and COVID-19 vaccines in the US, possibly reflecting COVID-19 vaccine safety concerns. Although the most recent data from the Centers for Disease Control (CDC) [10] indicates that $63.0 \%$ of individuals who received COVID-19 vaccines from December 14, 2020 to January 14, 2021 were female, this is likely due to the high prevalence of female healthcare professionals [11] and female nursing home residents [12]. Additionally, women are generally more likely to seek and receive healthcare than men [13]. Therefore, these data do not permit assessing the potential impact of COVID-19 vaccine misinformation on vaccine acceptance among women. Careful surveillance for such an impact is warranted, especially as COVID-19 vaccines become more widely available.

Recognizing the potential impact this misinformation may have had on patients deciding whether to receive COVID-19 vaccination will help clinicians ensure that such decisions are adequately informed. In the absence of safety and efficacy data regarding COVID-19 vaccines among pregnant and lactating patients, risk and benefit assessment of vaccination should be tailored to the individual. According to the CDC, observational data suggests that pregnant patients with COVID-19 are at increased risk for severe illness compared with nonpregnant individuals [14]. Moreover, COVID-19 related illness during pregnancy may increase the risk of pregnancy and birth complications [3]. Thus, vaccinating pregnant patients may prevent more severe COVID-19 related illness and pregnancy complications, as long as safety and efficacy are retained. Clinical trials assessing the safety and efficacy of COVID-19 vaccines in pregnant patients are currently in development or already under way. Regarding lactation, evidence shows that non live vaccines are safe for lactating individuals, and therefore, COVID-19 vaccines are thought to carry no risk to breastfeeding infants $[3,14]$.

The American College of Obstetricians and Gynecologists (ACOG) currently recommends that COVID-19 vaccines not be withheld from pregnant patients who meet criteria for vaccination based on priority groups recommended by the Advisory Committee on Immunization Practices (ACIP) and those at increased risk for COVID-19 acquisition, such as women healthcare workers [15]. ACOG also recommends that COVID-19 vaccines should be offered to lactating patients in a similar fashion to nonlactating individuals when they meet ACIP criteria [15]. ACOG also stated that excluding pregnant individuals from COVID-19 vaccine eligibility would violate their bodily autonomy and put them at risk of adverse outcomes and mortality stemming from COVID-19 related illness [16]. The American Society for Reproductive Medicine echoed the stance of ACOG, endorsing the recommendation for pregnant patients or those contemplating pregnancy to receive COVID-19 vaccination to mitigate risk of poorer outcomes [17]. We recommend that pregnant patients discuss COVID-19 vaccine decisions with their primary care physicians and obstetricians, and that physicians support patients in their personal decision to receive or refuse COVID-19 vaccines.

\section{Limitations}

A limitation of this study is that it is correlational and could not assess causality. Further studies are needed to investigate whether misinformation stemming from the December 2020 petition will impact vaccine hesitancy and acceptance by pregnant patients or those seeking to become pregnant. The nature of our study precludes it from 


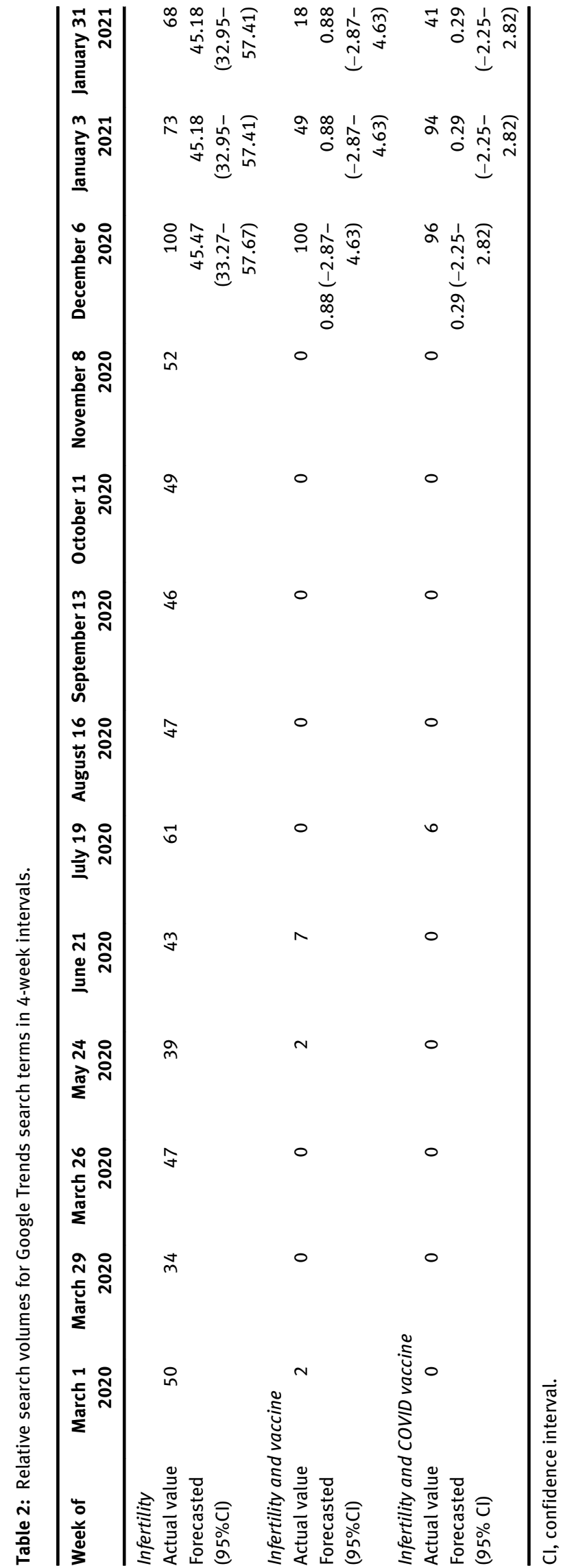

claiming causality and results should be interpreted accordingly.

\section{Conclusions}

Our results showed significant increases in internet searches for topics related to infertility and COVID-19 vaccines following misinformation about a December 2020 petition regarding safety and efficacy data for the BNT162b2 COVID-19 vaccine manufactured by BioNTech and Pfizer. The influence of COVID-19 vaccine misinformation on public perception may lead to changes in medical decision making. It is the duty of physicians and healthcare professionals to understand patient concerns, to be prepared with evidenced based information to help answer questions, and to assist patients in medical decision making. Dispelling misinformation and informing patients about the risks and benefits of COVID-19 vaccination may prevent unnecessary vaccine hesitancy or refusal, contributing to more successful vaccination efforts.

Research funding: None reported.

Author contributions: All authors provided substantial contributions to conception and design, acquisition of data, and analysis and interpretation of data; all authors drafted the article or revised it critically for important intellectual content; all authors gave final approval of the version of the article to be published; and all authors agree to be accountable for all aspects of the work in ensuring that questions related to the accuracy or integrity of any part of the work are appropriately investigated and resolved.

Competing interests: Dr Vassar has received grant funding from the National Institutes of Health, the U.S. Office of Research Integrity, and Oklahoma Center for the Advancement of Science and Technology for other research. Micah Hartwell has received research support through the US Department of Justice. Funding and support is unrelated to the current work.

Ethical approval: The Oklahoma State University Institutional Review Board determined that this study did not qualify as human subjects research.

\section{References}

1. Wodarg W, Scarlattilaan D. Petition/motion for administrative/ regulatory action regarding confirmation of efficacy end points and use of data in connection with the following clinical trial (s): 
phase III-eudract number: 2020-002641-2. corona-ausschuss.de. https://www.wodarg.com/app/download/9033912514/

Wodarg_Yeadon_EMA_Petition_Pfizer_Trial_FINAL_ 01DEC2020_signed_with_Exhibits_geschwa\%CC\%88rzt.pdf? $\mathrm{t}=1606870652$.

2. Gong R, Peng X, Kang S, Feng H, Huang J, Zhang W, et al. Structural characterization of the fusion core in syncytin, envelope protein of human endogenous retrovirus family W. Biochem Biophys Res Commun 2005;331:1193-200.

3. CDC. Vaccination considerations for people who are pregnant or breastfeeding. Published February 12, 2021. Available from: https://www.cdc.gov/coronavirus/2019-ncov/vaccines/ recommendations/pregnancy.html [Accessed 4 Mar 2021].

4. Kreis N-N, Ritter A, Louwen F, Yuan J. A message from the human placenta: structural and immunomodulatory defense against SARS-CoV-2. Cells 2020;9. https://doi.org/10.3390/ cells9081777.

5. Pinho AC. EMA recommends first COVID-19 vaccine for authorisation in the EU. Published December 21, 2020. Available from: https://www.ema.europa.eu/en/news/emarecommends-first-covid-19-vaccine-authorisation-eu [Accessed 4 Feb 2021].

6. Office of the Commissioner. Pfizer-BioNTech COVID-19 vaccine. Published March 2, 2021. Available from: https://www.fda.gov/ emergency-preparedness-and-response/coronavirus-disease2019-covid-19/pfizer-biontech-covid-19-vaccine [Accessed 4 Mar 2021].

7. FDA authorizes Moderna COVID-19 vaccine. Med Lett Drugs Ther 2021;63:9-10. Available from:https://www.ncbi.nlm.nih.gov/ pubmed/33512345.

8. Rodriguez A. No, the COVID-19 vaccine doesn't cause infertility in women. USA Today. Published December 10, 2020. Available from: https://www.usatoday.com/story/news/health/2020/12/10/ covid-vaccine-debunking-claims-causes-infertility-sterilization/ 6497018002/ [Accessed 4 Feb 2021].

9. Ayers JW, Leas EC, Johnson DC, Poliak A, Althouse BM, Dredze M, et al. Internet searches for acute anxiety during the early stages of the COVID-19 pandemic. JAMA Intern Med 2020;180: 1706-7.
10. Painter EM, Ussery EN, Patel A, Hughes MM, Zell ER, Moulia DL, et al. Demographic characteristics of persons vaccinated during the first month of the COVID-19 vaccination program - United States, December 14, 2020-January 14, 2021. Morb Mortal Wkly Rep 2021;70:174-7.

11. COVID-19 risks and impacts among health care workers by race/ ethnicity. Published November 11, 2020. Available from: https:// www.kff.org/racial-equity-and-health-policy/issue-brief/covid19-risks-impacts-health-care-workers-race-ethnicity/ [Accessed 4 Mar 2021].

12. Harris-Kojetin LD, Sengupta M, Lendon JP, Rome V, Valverde R, Caffrey C. Long-term care providers and services users in the United States, 2015-2016. Published Online February 2019. Available from: https://stacks.cdc.gov/view/cdc/76253 [Accessed 4 Mar 2021].

13. Owens GM. Gender differences in health care expenditures, resource utilization, and quality of care. J Manag Care Pharm 2008;14:2-6.

14. Interim clinical considerations for use of COVID-19 vaccines. Published March 4, 2021. Available from: https://www.cdc.gov/ vaccines/covid-19/info-by-product/clinical-considerations.html [Accessed 4 Mar 2021].

15. Vaccinating pregnant and lactating patients against COVID-19. Published February 4, 2021. Available from: https://www.acog. org/clinical/clinical-guidance/practice-advisory/articles/2020/ 12/vaccinating-pregnant-and-lactating-patients-against-covid19 [Accessed 13 Feb 2021].

16. Maternal immunization task force and partners urge that COVID-19 vaccine be available to pregnant individuals. Available from: https://www.acog.org/news/news-releases/2021/02/maternalimmunization-task-force-and-partners-urge-that-covid-19-vaccinebe-available-to-pregnant-individuals [Accessed 4 Mar 2021].

17. Update no. 12-testing and vaccine truths: ASRM patient management and clinical recommendations during the Coronavirus (COVID-19) pandemic. Am Soc Reprod Med. Published January 18, 2021. Available from: https://www.asrm. org/news-and-publications/news-and-research/press-releasesand-bulletins/update-no.-12-testing-and-vaccine-truths-asrmpatient-management-and-clinical-recommendations-during-thecoronavirus-covid-19-pandemic/ [Accessed 5 Mar 2021]. 